\title{
Bibliometric profile of research on antibiotics in sediments during 1958-2016
}

\author{
Chun-Li Zheng, Yi-Fei Wang, Qian-Ling-Lin Qiu, Gang Li* and Yao-Yang Xu
}

Antibiotic residues and antibiotic resistance genes (ARGS) can be accumulated in sediments. In this study, an improved bibliometric analysis was used to evaluate publications on antibiotics in sediments from the aspects of major countries, subject categories and keywords during 1958-2016. The results indicate that USA and China dominated studies in the recent period from 2005 to 2016. The most representative journal and subject category were Applied and Environmental Microbiology and Environmental Science and Ecology respectively. ARGs and antibiotic resistance bacteria involving mechanism such as sorption, adsorption and biodegradation were the mainstream research areas.

Keywords: Antibiotics, international collaborations, research hotspots, sediments, science categories.

ANTIBIOTICs have been mainly used for preventing diseases in humans and animals ${ }^{1}$. Globally, about 100,000 $200,000 \mathrm{t}$ of antibiotics is consumed each year, of which almost 25,000 $\mathrm{t}$ is used in China ${ }^{2}$. Most antibiotics are used to increase production as feed additives in aquaculture and fish farms ${ }^{3}$. Antibiotics can only be partially metabolized by humans and animals ${ }^{4}$. A significant amount of the non-metabolized portion is excreted along with urine and faeces, and enters the aquatic environment ${ }^{5}$. Wastewater treatment plants (WWTPs) present additional sources and are not designed to remove antibiotics from effluents ${ }^{6}$. Release of antibiotics into the environment leads to antibiotic residues in various water bodies, soils and sediments ${ }^{7,8}$.

Antibiotic residues may produce potential negative effects, which contribute to the selective pressure of microbial community in the environment ${ }^{9,10}$. The occurrence and dissemination of antibiotic resistance bacteria (ARB) will eventually threaten human health ${ }^{11-13}$. This is because they are resistant to high doses of some drugs and even there existed some superbug ${ }^{14}$. Noticeably, the existence of antibiotic resistance genes (ARGs) greatly increased the threat to humans by horizontal gene transfer

\footnotetext{
Chun-Li Zheng, Qian-Ling-Lin Qiu, Gang Li and Yao-Yang Xu are in the Key Laboratory of Urban Environment and Health, Institute of Urban Environment, Chinese Academy of Sciences, Xiamen 361021, People's Republic of China; Chun-Li Zheng and Qian-Ling-Lin Qiu are also in the University of Chinese Academy of Sciences, Beijing 100049, People's Republic of China; Chun-Li Zheng, Qian-Ling-Lin Qiu, Gang Li and Yao-Yang Xu are also in Ningbo Key Laboratory of Urban Environment Processes and Pollution Control, Ningbo Urban Environment Observation and Research Station, Chinese Academy of Sciences, Ningbo 315800, People's Republic of China; Yi-Fei Wang is in the Civil and Environmental Engineering Department, University of Houston, Houston 77054, the United States of America.

*For correspondence. (e-mail: gli@iue.ac.cn)
}

$(\mathrm{HGT})^{15,16}$. Owing to the physico-chemical properties of antibiotics, they can easily accumulate in sediments. Also, sediments are regarded as an important reservoir of antibiotics in the environment ${ }^{17}$. Some studies have shown that the concentration of antibiotics in sediments varies over a wide range (from $\mathrm{ng} / \mathrm{g}$ to $\mu \mathrm{g} / \mathrm{g}$ ) (Table 1 ). The concentration of norfloxacin in Hai River ranged from 5.95 to $5770 \mathrm{ng} / \mathrm{g}$ (ref. 10). Feitosa-Felizzola and Chiron $^{18}$ reported that clarithromycin and azithromycin were ubiquitous in sediments. A study of 12 selected antibiotics in the sediment samples of Liao River in China showed that ofloxacin, oxytetracycline and norfloxacin were the prominent antibiotics in sediments, with mean concentration of $152.2 \pm 108.3,149.5 \pm 147.6$ and $62.8 \pm$ $83.3 \mathrm{ng} / \mathrm{g}$ respectively ${ }^{19}$.

Bibliometric analysis has been broadly applied to quantitatively analyse publications and predict the development trends of a certain field using abundant data ${ }^{20}$. Several researchers have used bibliometric analysis in the fields such as medical research, biological resources,

Table 1. Concentration of detected antibiotics in sediment samples around the world

\begin{tabular}{llc}
\hline Sediments of rivers and lakes & Concentration $\left(\mathrm{ng} \mathrm{g}^{-1}\right)$ & Reference \\
\hline Liao River, China & ND-1236.9 & 19 \\
Wangyang River, China & ND-186218.5 & 44 \\
Nanming River, China & ND-1780 & 45 \\
Taihu Lake, China & ND-43.58 & 46 \\
Zizhuyuan Lake, China & ND-94.97 & 47 \\
Lambro River, Italy & ND-6.3 & 48 \\
Ebro River, Spain & ND-131 & 49 \\
Poudre River, USA & ND-62.6 & 16 \\
Arc River, France & ND-267.42 & 18 \\
\hline
\end{tabular}

ND, Not detected. 
environmental protection, etc. ${ }^{21-24}$. Science Citation Index (SCI) database of the Web of Science (WoS) is one of the most popular and widely used data source of bibliometric information to extract high-quality articles. SCI is also used to evaluate the development, collaborations, hotspots and future research directions ${ }^{25}$. Likewise, network analysis, a new tool in statistical analysis, can visualize connections between countries and subject categories simultaneously ${ }^{26}$. Revealing future research directions, keywords analysis can provide important information about research trends and hotspots. However, no publications are available on the studies of antibiotics in sediments using the bibliometric method.

Previous studies primarily focused on the aquatic systems and soils. Therefore, this study presents an overview of the research on antibiotics in sediments during the period from 1958 to 2016. Hierarchical cluster analysis (HCA) was used to describe the research status in a novel way as a combination of author addresses, subject categories and author keywords ${ }^{27}$. This study principally included three aspects of antibiotics in sediments as follows: (1) analysis of progress regarding international collaborations in different stages among the 20 most productive countries using HCA and network analysis; (2) ranking the most popular journals with publication and visualization of the variation in subject categories among the 20 most prolific disciplines of antibiotics in sediments; and (3) extraction of the top 50 keywords to confirm research trends and hotspots.

\section{Materials and methods}

We generated a bibliometric dataset of antibiotics in sediments on 8 April 2017. Data were downloaded from SCI database of the Web of Science Core Collection (Clarivate Analytics). We chose 'antibiotics' as the topic and used 'sediment' or 'sediments' to refine advance search results between the years 1900 and 2016. The time from 1900 to 2016 is the collection time of WoS. Full records, including author addresses (C1), published year (PY), subject categories (SC), author keywords (DE), journal name $(\mathrm{JN})$ and cited documents $(\mathrm{CD})$ were considered and downloaded. We analysed a total of 1206 publications related to antibiotics in sediments. Owing to the absence of some information of $\mathrm{C} 1, \mathrm{SC}$, and $\mathrm{DE}$ among some early publications, the starting times of C1 (1973), SC (1958), and DE (1991) were different. Further, data were preprocessed by merging the keywords with similar meanings, and ranked from the most to least frequently appearing keywords. Articles from England, North Ireland, Scotland and Wales were reclassified as publications of the United Kingdom. Articles from Taiwan were also merged into one group under China.

Using the PCORD 4.0 software, we grouped all countries, all subject categories, and 200 most frequently appearing keywords into stages (time-periods) by HCA under a uniform yet analogous level. Next, the Bibexcel and Pajek software were used to rank the most commonly used journals for publication. We also visualized the relationships among 20 most publication-productive countries, 20 most prolific subject categories, and 50 most frequently appearing keywords ${ }^{28-30}$.

Using the visualization network, we observed the changes and compared the network of countries, subject categories and keywords step by step. Microsoft Excel 2010 and SigmaPlot 12.5 software were used to perform statistical analysis of publications and publication times for countries, subject categories and keywords. The publication times for countries, subject categories and keywords were computed as

$$
\text { Publication time }=\sum_{i=1}^{N} \text { Number of different 'countries' }
$$$$
\text { per paper, }
$$

where $N$ is the number of papers, and 'subject' refers to the category of interest, namely countries, subject categories and keywords. Example calculations are presented in the Supplementary Table 1.

\section{Results and discussion}

\section{Progress of international collaborations}

Author addresses were used in the analysis of research findings at the international level. From 1173 articles, which accounted for $97.3 \%$ of the total 1206 articles, author addresses were obtained during 1973 to 2016 . The results yielded a total of 79 countries that produced precisely 1479 publication times. Among these, three countries $(3.8 \%)$ produced only one publication time and $7(8.9 \%)$ published less than ten publication times. On the other hand, each of 20 most publicationproductive countries $(25.3 \%)$ yielded more than 13 publication times during the same period, contributing a total of 1413 publication times $(95.5 \%$ of total 1479 publication times).

Cluster analysis was performed using publication times for all countries, science categories and keywords (Figure 1). In order to observe the changes in international collaborations over time, the historical research efforts were grouped into three main clusters (I-III). These clusters were divided into three main stages on the basis of timeperiod: stage I (1973-1990), stage II (1991-2004), and stage III (2005-2016) (Figure 2). Figure 2 shows that the number of publications on antibiotics in sediments was low during stage I, and began to increase during stage II, especially for USA followed by China. In stage III (after the year 2011), more articles were published by China 
than USA. Likewise, analysis of the 20 most productive countries was performed for each of the three stages based on collaborations between the countries for publications on antibiotics in sediments (Figure 3 ). Figure 3 highlights the progress of international collaborations to gain and share scientific knowledge for a better understanding of antibiotics in sediments at the international level.

Stage I (covering 18 years) is the earliest research stage (Figure $3 a$ ). As shown in Figure $3 a$, the publication output in stage I is low (one article per year on
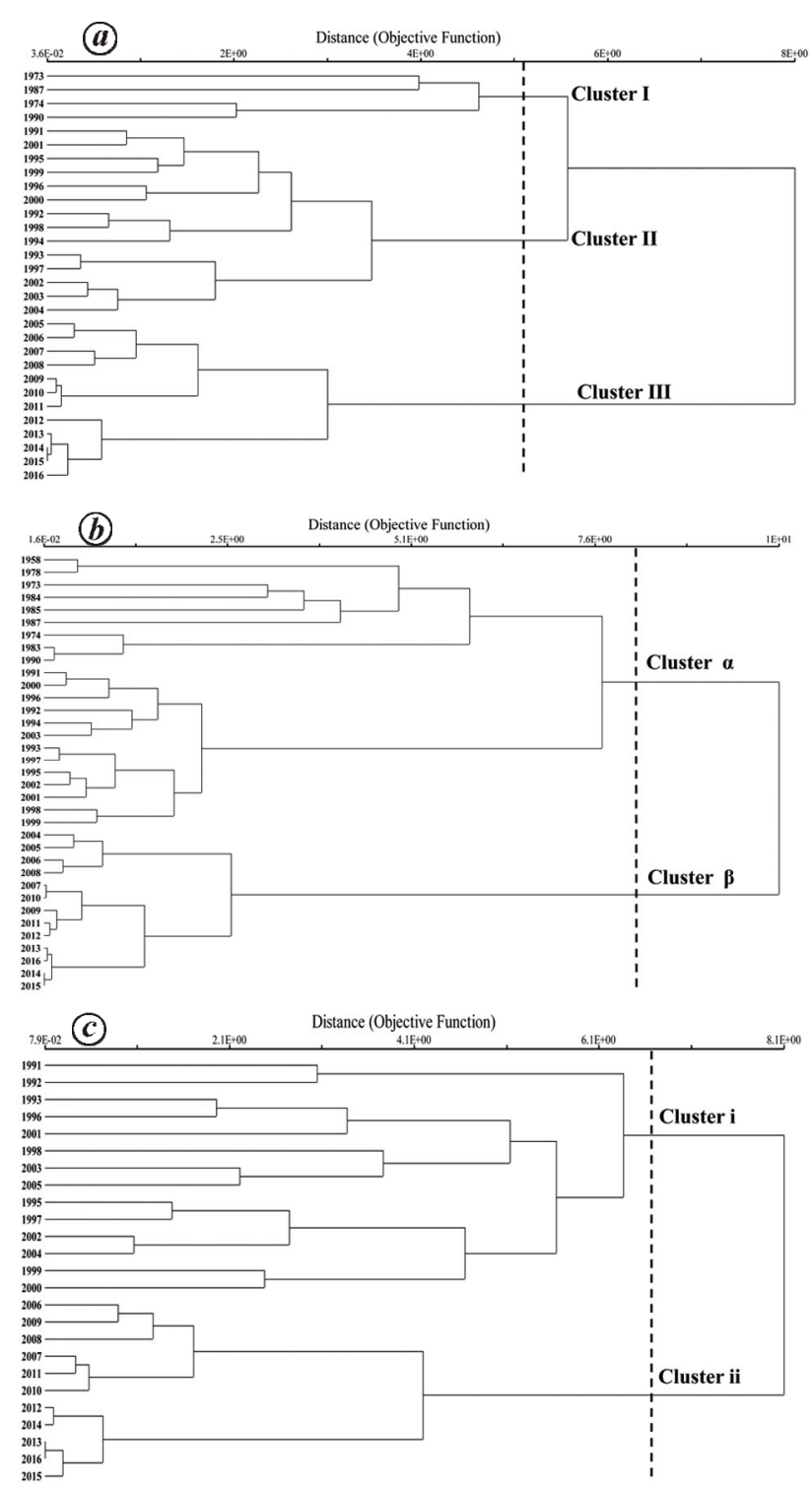

Figure 1. Dendrograms of several stages of research efforts relative to progress of $(\boldsymbol{a})$ international collaborations; $(\boldsymbol{b})$ variation in subject categories; $(c)$ research trends and hotspots based on all the countries, subject categories and the 200 most frequently appearing keywords in articles on antibiotics in sediment research.

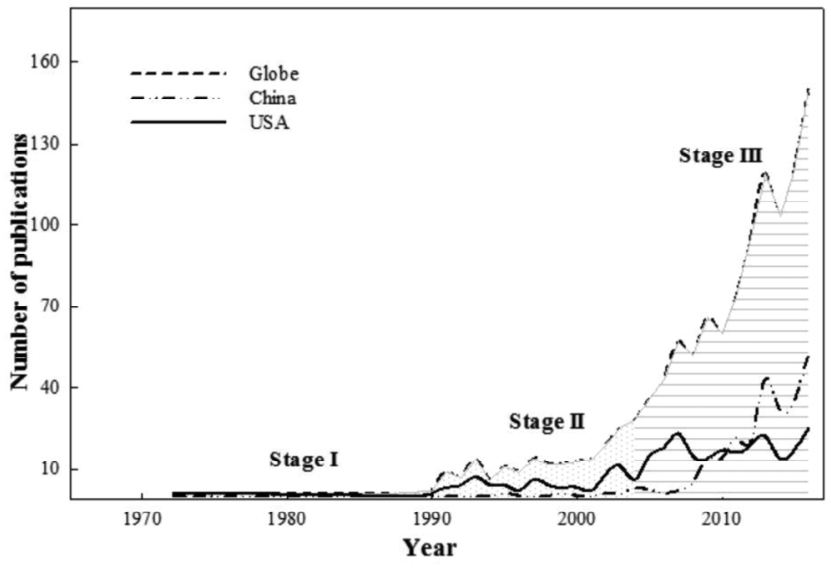

Figure 2. Number of articles on antibiotics in sediment research published annually around the world, China and the United States during 1973-2016 in three different stages (I-III).

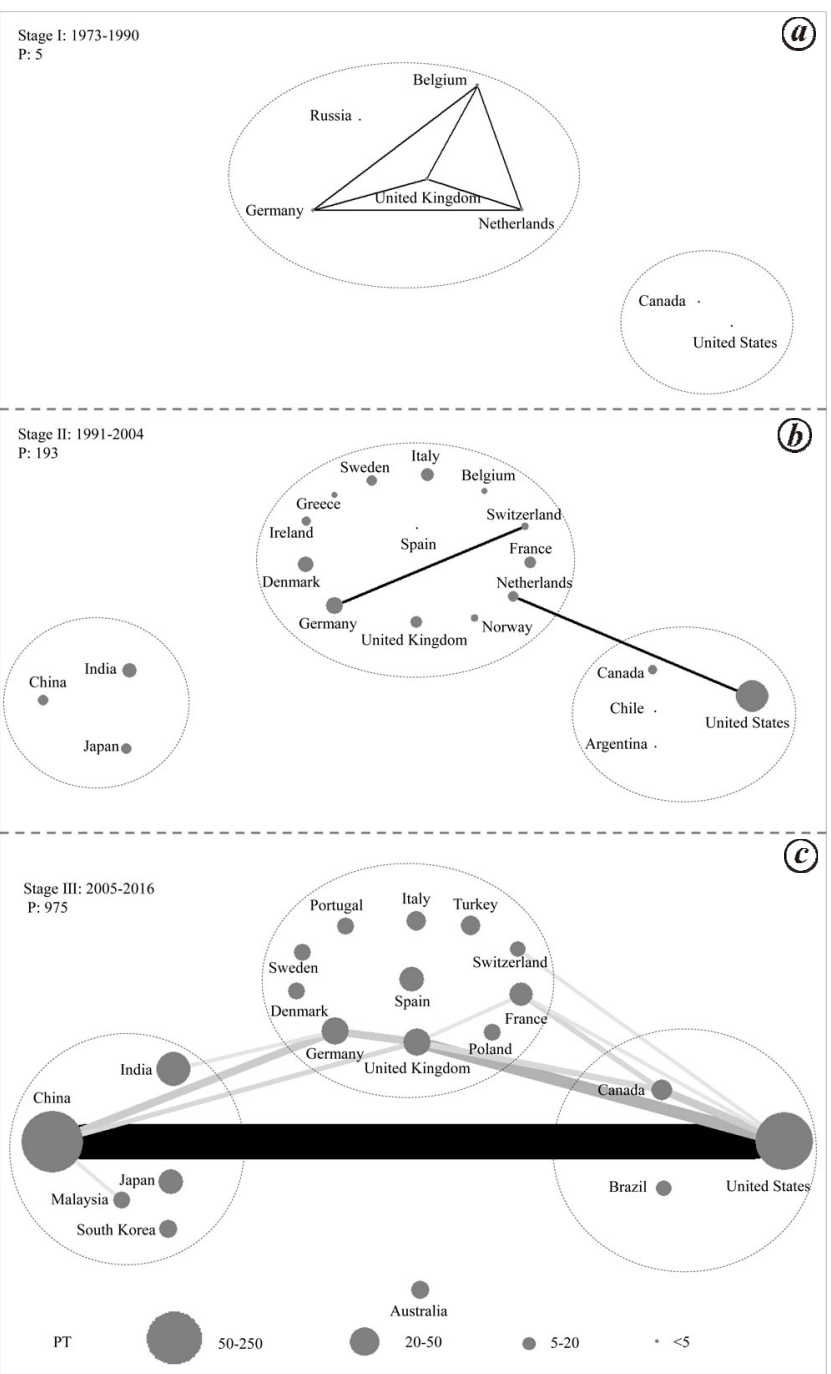

Figure 3. Collaboration networks for the 20 most productive countries, visualized at $(\boldsymbol{a})$ stage I, $(\boldsymbol{b})$ stage II and $(\boldsymbol{c})$ stage III based on publication time of each country on antibiotics in sediment research. $\mathrm{P}$ and PT refer to the number of publications and publication time of each stage respectively. 
average), with a total of five articles that account for only $0.4 \%$ of all 1173 articles. Stage I is preliminary period of research on antibiotics in sediments, having the characteristics of lowest output and longest duration. In this stage, authors were affiliated with seven countries (including territories). The total five articles had 33 publication times, accounting for $0.5 \%$ of 1479 publication times. As shown the network analysis of the most productive countries, showed collaborations from co-affiliated institutional relationships of the United Kingdom, Germany, Belgium and the Netherlands in stage I. The seven countries are from two continents; the UK, Germany, Russia, Belgium and the Netherlands are from Europe, while USA and Canada are from North America.

The research efforts on antibiotics in sediments proliferated during stage II (Figure $3 b$ ). The publication output increased dramatically to more than 14 articles per year, amounting to 193 articles, which is $16.5 \%$ of the total 1173 articles. The author addresses (obtained from all 193 articles) showed 34 countries, with 2311 publication times, corresponding to $15.6 \%$ of the total of 1479 publication times. These data showed that the research efforts on antibiotics in sediments started to develop rapidly. Thus, stage II can be categorized as a fast-developing stage. In stage II, the 20 most publication-productive countries altogether generated 213 publication times (92.3\% of the total 2311 publication times). The network analysis further revealed that the international collaborations increased among the countries located in Europe (Norway, Switzerland, France, Spain, Italy, Sweden, Greece, Ireland and Denmark) and America (Chile and Argentina). Some countries in Asia such as China, Japan and India appeared among the 20 most productive countries/territories in this stage. Notably, international collaborations were extensive than those in stage I among countries in America and Europe.

The most productive efforts were made during stage III, evidenced by the rapid increase in publication output (Figure $3 c$ ). This stage, more than 82 articles were published each year by 76 countries, totalling 975 articles, accounting for $83.1 \%$ of the total 1173 articles. In other words, 76 countries produced 1240 publication times, corresponding to $83.8 \%$ of the total of 1479 publication times. The top 20 most productive countries published an accumulative combined total of 998 publication times, accounting for $80.5 \%$ of the total 1240 publication times. The network analysis showed that international collaborations in this stage included more countries located in Europe (Poland, Portugal and Turkey), America (Brazil) and Asia (Malaysia and South Korea) compared to stages I and II. Noticeably, Australia made its first appearance among the 20 most productive countries. Stage III can be interpreted as an active developing stage. The cooperation was strongest between USA and China. In stage III, the international collaborations of antibiotics in sedi- ments became much more extensive and stronger than other stages. China did not only collaborate with USA, but expanded its collaborations to other countries like Germany, UK and Malaysia, with an increasing trend. Adding to the results, international institutional collaborations of countries within Europe and America became much stronger in this stage than in stages I and II. However, the fact to be considered is that research collaborations were fewer among countries in Asia; thus more efforts should be made in this direction by including more countries from Asia.

\section{Journals and variation of subject categories}

In this study, all articles were obtained from 407 journals belonging to a variety of disciplines in the field of antibiotics in sediments. Among them, 265 journals published only one article on this subject while 379 journals published less than 10 articles $(93.1 \%$ of all 407 journals). However, the most frequently used 20 journals published a total of 469 articles, accounting for $38.9 \%$ of all 1206 articles (Table 2). Among these journals, Applied and Environmental Microbiology published the most number of articles (53; accounting for $4.39 \%$ of the total of 1206 articles) and had the highest $h$-index of 29. Environmental Science and Technology ranked second with 49 articles published; the number of total citations and average citations were 2952 and 60.24 respectively. Chemosphere which had the highest average citations of 87.23 and Science of the Total Environment which published 35 articles $(2.9 \%$ of the total 1206 articles) ranked third and fourth respectively. Water Research had the highest impact factor of 5.99 and ranked first in the category of 'water resources' $(G)$ second in the category of 'engineering, environmental' (C) and seventh in the category of 'environmental sciences' (D).

During the publication period from 1958 to 2016, there were 63 different subject categories, corresponding to $99.9 \%$ of all 1206 articles and equivalent to 2009 publication times. Using cluster analysis, the 63 subject categories were grouped into two main clusters stages ( $\alpha$ and $\beta$ ) based on publication year. The network analysis was performed for 20 major subject categories in each of these two stages (Figure 4). Here, an attempt was the variation in subject categories helped us acquire and share scientific knowledge for a better understanding of the collaborative research efforts.

In stage $\alpha$ (1958-2003), 201 articles on antibiotics in sediments were published, accounting for $16.7 \%$ of all 1205 articles, and equivalent to 351 publication times (Figure $4 a$ ). The 20 most prolific subject categories were grouped into seven main sections that represent a relationship between different subject categories. First, 'Environmental sciences and ecology' was a comprehensive subject to examine all environment-related issues and 
Table 2. Twenty most popular journals with the number of publications, citations, impact factors, $h$-index and Web of Science (WoS) category

\begin{tabular}{rrrrrrll}
\hline JR & TP & TP $(\%)$ & TC & TC/TP & IF & $h$-index & \multicolumn{1}{l}{ WoS category } \\
\hline 1 & 53 & 4.39 & 2519 & 47.53 & 3.82 & 29 & A $(33 / 161) ; \mathrm{B}(31 / 123)$ \\
2 & 49 & 4.06 & 2952 & 60.24 & 5.39 & 27 & C $(3 / 50) ; \mathrm{D}(14 / 225)$ \\
3 & 40 & 3.32 & 3489 & 87.23 & 3.70 & 24 & $\mathrm{D}(37 / 225)$ \\
4 & 35 & 2.90 & 1903 & 54.37 & 3.98 & 16 & $\mathrm{D}(32 / 225)$ \\
5 & 30 & 2.49 & 785 & 26.17 & 3.10 & 11 & $\mathrm{D}(56 / 225) ; \mathrm{E}(6 / 104)$ \\
6 & 27 & 2.24 & 1132 & 41.93 & 1.89 & 18 & $\mathrm{~F}(14 / 52) ; \mathrm{E}(38 / 104)$ \\
7 & 23 & 1.91 & 145 & 6.30 & 2.76 & 7 & $\mathrm{D}(65 / 225)$ \\
8 & 22 & 1.82 & 1197 & 54.41 & 5.99 & 15 & $\mathrm{C}(2 / 50) ; \mathrm{D}(7 / 225) ; \mathrm{G}(1 / 85)$ \\
18 & 14 & 1.16 & 365 & 26.07 & 4.84 & 10 & $\mathrm{~N}(2 / 126) ; \mathrm{C}(7 / 50) ; \mathrm{D}(19 / 225)$ \\
19 & 13 & 1.08 & 692 & 53.23 & 3.93 & 9 & $\mathrm{O}(14 / 77) ; \mathrm{P}(11 / 75)$ \\
20 & 12 & 1.00 & 178 & 14.83 & 3.38 & 10 & $\mathrm{~A}(41 / 161)$ \\
\hline
\end{tabular}

Journals rank (JR): 1, Applied and Environmental Microbiology; 2, Environmental Science and Technology; 3 , Chemosphere; 4, Science of the Total Environment; 5, Marine Pollution Bulletin; 6, Aquaculture; 7, Environmental Science and Pollution Research; 8, Water Research; 9, Frontiers in Microbiology; 10, FEMS Microbiology Ecology; 11, PLoS ONE; 12, Journal of Natural Products; 13, Journal of Antibiotics; 14, Microbial Ecology; 15, Environmental Pollution; 16, Marine Drugs; 17, Journal of Applied Microbiology; 18, Journal of Hazardous Materials; 19, Journal of Chromatography A; 20, Applied Microbiology and Biotechnology.

WoS categories: A, Biotechnology and applied microbiology; B, Microbiology; C, Engineering, environmental; D, Environmental sciences; E, Marine and freshwater biology; F, Fisheries; G, Water resources; H, Multidisciplinary Sciences; I, Chemistry, medicinal; J, Pharmacology and pharmacy; K, Plant sciences; L, Immunology; M, Ecology; N, Engineering, civil; O, Biochemical research methods; P, Chemistry, analytical.

TP, Total publications; TC, Total citations; TC/TP, Average of citations per article; IF, Impact factor (2015).

discoveries. With more negative effects due to the abuse of antibiotics, 'Chemistry' and 'Toxicology' were important subjects in order to explore the mechanism of antibiotics in sediments. The discipline 'Engineering' provided some practical measures to solve problems. 'Microbiology' was the core discipline related to biology, including 'Biotechnology and applied microbiology', 'Biochemistry and molecular biology', and 'Marine and freshwater biology'. The disciplines in the first section had some connections with the subject categories in medicine and health consisting of 'Pharmacology and pharmacy', 'Veterinary sciences', 'Infectious diseases', 'Immunology', 'General and internal medicine' and 'Public, environmental and occupational health', which showed that the field of antibiotics in sediments is mainly related to the field of medicine. Secondly, the sixth section consisted of 'Water resources' and 'Oceanography' which had less connection with water research, while 'Geology' had some connection with water research. Finally, 'Agriculture', 'Fisheries' and 'Plant sciences' focused mainly on food safety.

In stage $\beta$ (2004-2016), the number of articles in the field of antibiotics in sediments was 1004, accounting for $83.3 \%$ of all 1205 articles leading to 1685 publication times. The result indicated that the importance of research on this topic was well recognized in stage $\beta$ (Figure $4 \mathrm{~b}$ ). Not only did the number of articles increase significantly, but also the connections among the disciplines increased. 'Science and technology - other topics' was an emerging subject in the technical part, which showed that there were some new solutions to deal with problems caused by abuse of antibiotics with engineering measures in this stage. Moreover, 'Food science and technology' was part of food safety in the top 20 most prolific subjects, which showed that people paid more attention to it. Noticeably, the relationship among the seven sections of subject categories was much stronger than in stage $\alpha$, such as among 'Microbiology', 'Biotechnology and applied microbiology', 'Engineering' and 'Environmental sciences and ecology.' Especially, there were two obvious pairs, viz. 'Microbiology' with 'Biotechnology and applied microbiology' and 'Engineering' with 'Environmental sciences and ecology', which indicated that antibiotics in sediments research began to contain more comprehensive studies.

\section{Research tendencies and hotspots}

In the field of antibiotics in sediments, the total number of articles during the publication period from 1991 to 2016 was 861, which accounted for $71.4 \%$ of all 1206 articles, and was equivalent to 4394 publication times. In these 861 articles, 2406 unique keywords were obtained, of which 1948 keywords appeared only once while 2356 keywords appeared less than 10 times, corresponding to $81.0 \%$ and $97.9 \%$ of the total 2406 keywords respectively. We determined 200 most frequently used keywords by HCA, and then grouped them into two stages (i and ii) from 1990 to 2016. Figure 5 shows the relationships of 50 most frequently used keywords in various stages using the co-words network. During these two stages, 'Antibiotics' was cited most as a keyword. 


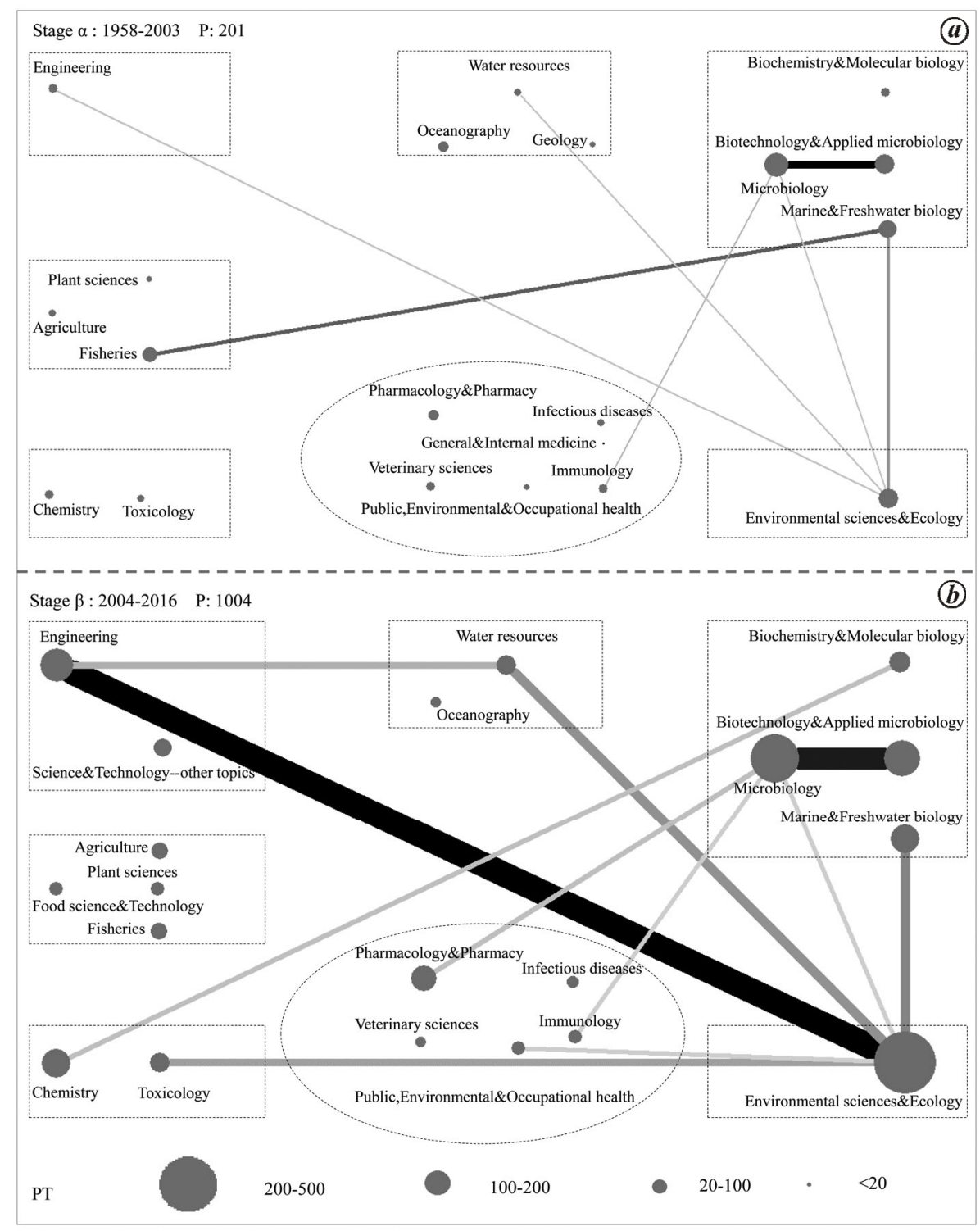

Figure 4. Variation in the 20 most frequent subject categories visualized at $(\boldsymbol{a})$ stage $\alpha$ and $(\boldsymbol{b})$ stage $\beta$ based on publication time of each subject category on antibiotics in sediment research. P and PT refer to the number of publications and the publication times of each stage respectively.

In stage $i$, a total of 132 articles were published during the period 1991-2005, accounting for $15.3 \%$ of all 861 publications, and 667 publication times (Figure $5 a$ ). There were eight parts focused on research regarding antibiotics in sediments. 'Fluoroquinolones', 'Tetracycline', 'Oxytetracycline', 'Flumequine' and 'Oxolinic acid' were the commonly used antibiotics in this stage ${ }^{31,32}$. As 'Pharmaceuticals' for humans, they can alleviate symptoms caused by infectious diseases. However, most of them were applied to 'Fish farm' and 'Aquaculture', including 'Shrimp', Penaeus monodon, 'Fontinalis antipyretica' and 'Atlantic salmon' in order to accomplish the 'Most probable number' for animals. The keywords 'Sediment', 'Wastewater', 'Soil', 'Marine' and 'Marine sediment' showed that there were some links between the distribution of antibiotics among 'Environment' and
'Marine environment' media ${ }^{33}$. Moreover, some related 'Environment analysis' and 'Water analysis' were required to evaluate the 'Environment effects'. The fifth section was about biology, which also showed some negative influences on the proliferation of antibiotics. With the appearance of 'Resistance', 'Residues', 'Antibiotic resistance' and 'Heavy metal resistance', many researchers began to focus on the sources, distribution and dissemination of antibiotics ${ }^{34}$. Some biological terms, including 'Xenobiotics', 'Microcosms', 'Rhizosphere', 'Plasmid', 'PCR' and 'Diversity' were applied to explore 'Antimicrobial activity' of antibiotics. Noticeably, 'Marine microorganisms', 'Bacteria' and 'Marine bacteria' that contained 'Streptomyces', 'Actinomycetes', Escherichia coli, 'Fish bacteriology', Pseudomonas and Bacillus were studied extensively in this area ${ }^{35-37}$. LC-MS/MS, 
GENERAL ARTICLES

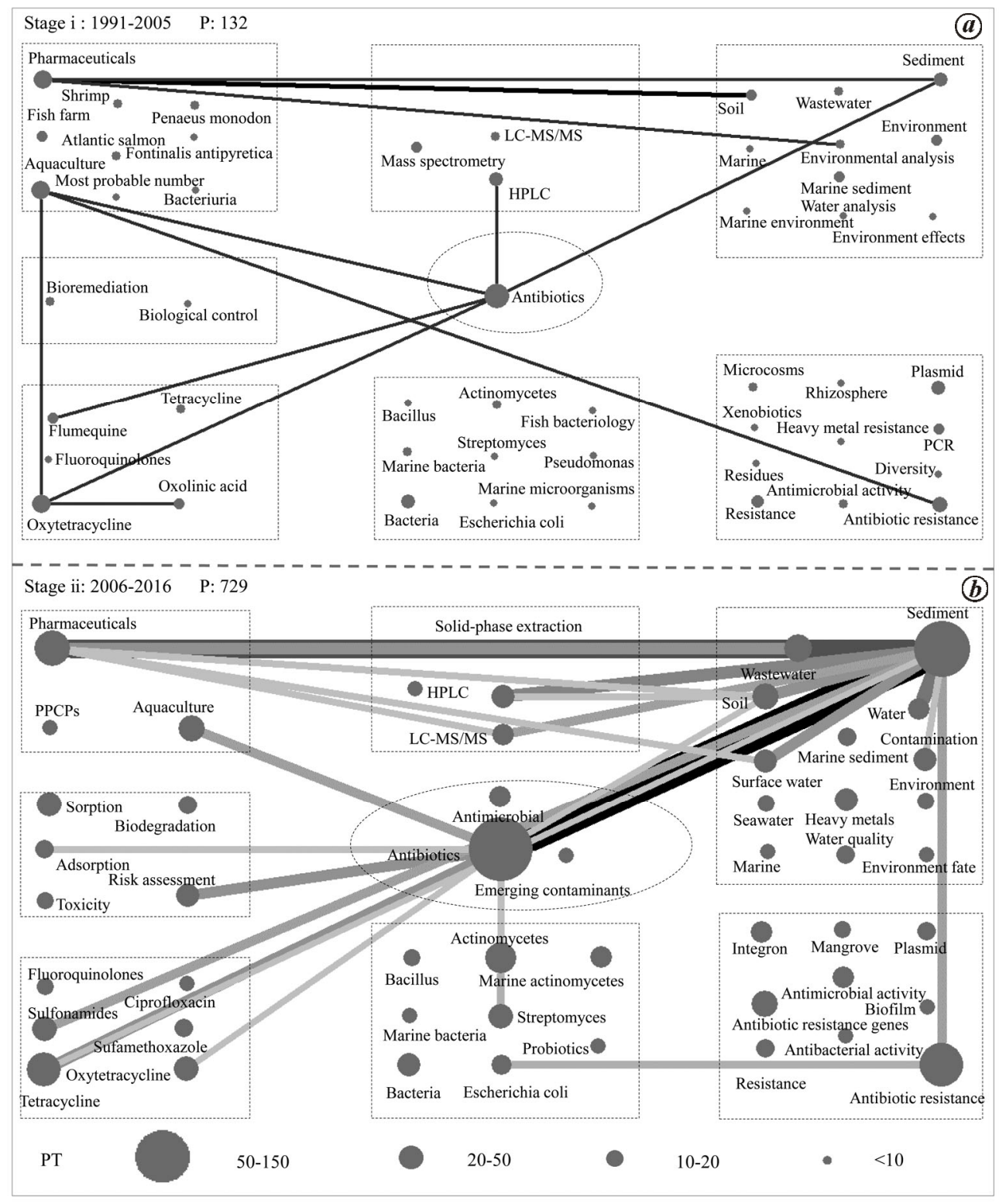

Figure 5. Relationship among the 50 most frequently used keywords visualized at (a) stage i and (b) stage ii based on publication frequency of each keyword on antibiotics in sediment research. $P$ refers to the number of publications for modelling network of each stage. PT refers to the publication time of each keyword among the top 50 keywords.

HPLC and 'Mass spectrometry' were the commonly used methods to detect antibiotics ${ }^{38}$. Finally, 'Bioremediation' and 'Biological control' were about mechanism researches of controlling the negative effects of antibiotics caused in this stage.

Stage ii lasted for almost 11 years, in which a total of 729 articles on antibiotics in sediments were published, corresponding to $84.7 \%$ of all 861 articles and equivalent to 3727 publication times (Figure $5 \mathrm{~b}$ ). 'Antimicrobial' was a kind of antibiotic and antibiotics were described as 'Emerging contaminants' in this stage. 'Tetracyclines', 'Fluoroquinolones' and 'Sulfonamides', including 'Tetracycline', 'Oxytetracycline', 'Ciprofloxacin' and 'Sulfa- methoxazole' were the frequently used classes of antibio$\operatorname{tics}^{39}$. They were mainly applied to 'Pharmaceuticals', 'Pharmaceutical and personal care products (PPCPs)' and 'Aquaculture'. Some reports indicated that there has been 'Contamination', such as 'Heavy metals' in sediments. Therefore, research on antibiotics in sediments expanded to include 'Water', 'Surface water' and 'Seawater'. Furthermore, 'Water quality' and 'Environment fate' were also studied thoroughly. 'Antibacterial activity' and 'Antibiotic resistance genes' were included in the top 50 keywords compared to stage $\mathrm{i}$ in the part of 'Biology', which showed that the negative effects of unreasonable use and disposal of antibiotics had increased. 
Particularly, some researchers started exploring relationships of 'Antibiotic resistance genes' with other related factors such as 'Integron', 'Mangrove' and 'Biofilm'. However, some kinds of 'Probiotics' have been selected against infectious diseases. Furthermore, 'Marine actinomycetes' appeared among the most studied classes of bacteria in this stage ${ }^{40}$. The appearance of 'Solid-phase extraction' showed that some innovative methods were being applied to qualitatively and quantitatively determine the concentration of antibiotics in sediments. 'LCMS/MS' was the most commonly used method in this pe$\operatorname{riod}^{41}$. More mechanism to reduce the threat caused by antibiotics were focused on 'Sorption', 'Adsorption', 'Toxicity' and 'Biodegradation' ${ }^{42}$. Additionally, 'Risk assessment' about antibiotics in sediments called for further studies in the future ${ }^{43}$.

\section{Conclusion}

This study presents an overview of antibiotics in sediments. We have performed a qualitative and quantitative analysis on the developments and trends in this field using an improved bibliometric analysis method. The results showed that China developed rapidly in the field of antibiotics in sediments and matched with USA until 2011. The analysis of 20 most publication-productive countries indicated that stage III (2005-2016) showed the fastest development and made the most achievements. The 20 most productive countries were mainly located in America (USA, Canada and Brazil), Europe (UK, Germany, France, Italy, Denmark, Sweden, Portugal, Poland, Turkey, Switzerland and Spain) and Asia (China, Japan, South Korea, Malaysia and India). Moreover, there were further collaborations within Europe and America.

Among the 20 most frequently used journals, Applied and Environmental Microbiology, Environmental Science and Technology and Chemosphere ranked first, second and third respectively. 'Environmental sciences and ecology' was the most studied subject category and there was a close connection between 'Engineering' and 'Environmental sciences and ecology' as well as between 'Microbiology' and 'Biotechnology and applied microbiology'. Owing to the unreasonable use of 'Antibiotics', they were regarded as 'Emerging contaminants'. 'Tetracycline', 'Oxytetracycline', 'Sulfamethoxazole' and 'Ciprofloxacin' were widely researched in the field of antibiotics in sediments. LC-MS/MS was the method principally used to study antibiotics. The research on antibiotics in sediments mainly focused on 'Wastewater' and 'Surface water'. Moreover, people began to worry about 'Antibiotic resistance' and 'Antibiotic resistance genes'. 'Actinomycetes' and Streptomyces were the main categories of 'Bacteria' in sediments. In order to explore effects like 'Toxicity' produced by the overuse of antibiotics, mechanisms including 'Sorption', 'Adsorption' and 'Biodegradation' were considered. Conclusively, it is suggested that antibiotics in sediments research should focus on 'Antibiotic resistance genes' in future.

1. Kummerer, K., Antibiotics in the aquatic environment-a review - part I. Chemosphere, 2009, 75, 417-434.

2. Zhang, C., Tang, J., Wang, L., Gao, X. and He, X., Occurrence of antibiotics in water and sediment from Zizhuyuan Lake. Pol. J. Environ. Stud., 2015, 24, 1831-1836.

3. Hirsch, R., Ternes, T., Haberer, K. and Kratz, K. L., Occurrence of antibiotics in the aquatic environment. Sci. Total Environ., 1999, 225, 109-118.

4. Sarmah, A. K., Meyer, M. T. and Boxall, A. B., A global perspective on the use, sales, exposure pathways, occurrence, fate and effects of veterinary antibiotics (VAs) in the environment. Chemosphere, 2006, 65, 725-759.

5. Kolpin, D. W., Furlong, E. T., Meyer, M. T., Thurman, E. M., Zaugg, S. D., Barber, L. B. and Buxton, H. T., Pharmaceuticals, hormones, and other organic wastewater contaminants in U.S. streams, 1999-2000: a national reconnaissance. Environ. Sci. Technol., 2002, 36, 1202-1211.

6. Hamscher, G., Sczesny, S., Hoper, H. and Nau, H., Determination of persistent tetracycline residues in soil fertilized with liquid manure by high-performance liquid chromatography with electrospray ionization tandem mass spectrometry. Anal. Chem., 2002, 74, 1509-1518.

7. Thiele-Bruhn, S., Pharmaceutical antibiotic compounds in soils a review. J. Plant Nutr. Soil Sci., 2003, 166, 145-167.

8. Kim, S. and Carlson, K., Temporal and spatial trends in the occurrence of human and veterinary antibiotics in aqueous and river sediment matrices. Environ. Sci. Technol., 2007, 41, 50-57.

9. Halling-Sørensen, B., Nors Nielsen, S., Lanzky, P. F., Ingerslev, F., Holten Lützhøft, H. C. and Jørgensen, S. E., Occurrence, fate and effects of pharmaceutical substances in the environment $-\mathrm{a}$ review. Chemosphere, 1998, 36, 357-393.

10. Zhou, L. J., Ying, G. G., Zhao, J. L., Yang, J. F., Wang, L., Yang, B. and Liu, S., Trends in the occurrence of human and veterinary antibiotics in the sediments of the Yellow River, Hai River and Liao River in northern China. Environ. Pollut., 2011, 159, 18771885.

11. Samuelsen, O. B., Torsvik, V. and Ervik, A., Long-range changes in oxytetracycline concentration and bacterial resistance toward oxytetracycline in a fish farm sediment after medication. Sci. Total Environ., 1992, 114, 25-36.

12. Baquero, F., Martinez, J. L. and Canton, R., Antibiotics and antibiotic resistance in water environments. Curr. Opin. Biotechnol., 2008, 19, 260-265.

13. Yang, J. F., Ying, G. G., Zhao, J. L., Tao, R., Su, H. C. and Chen, F., Simultaneous determination of four classes of antibiotics in sediments of the Pearl River using RRLC-MS/MS. Sci. Total Environ., 2010, 408, 3424-3432.

14. Martinez, J. L., Environmental pollution by antibiotics and by antibiotic resistance determinants. Environ. Pollut., 2009, 157, 2893-2902.

15. Cabello, F. C., Heavy use of prophylactic antibiotics in aquaculture: a growing problem for human and animal health and for the environment. Environ. Microbiol., 2006, 8, 1137-1144.

16. Pei, R., Kim, S. C., Carlson, K. H. and Pruden, A., Effect of river landscape on the sediment concentrations of antibiotics and corresponding antibiotic resistance genes (ARG). Water Res., 2006, 40, 2427-2435.

17. Capone, D. G., Weston, D. P., Miller, V. and Shoemaker, C., Antibacterial residues in marine sediments and invertebrates following chemotherapy in aquaculture. Aquaculture, 1996, 145, 5575 . 
18. Feitosa-Felizzola, J. and Chiron, S., Occurrence and distribution of selected antibiotics in a small Mediterranean stream (Arc River, Southern France). J. Hydrol., 2009, 364, 50-57.

19. Dong, D., Zhang, L., Liu, S., Guo, Z. and Hua, X., Antibiotics in water and sediments from Liao River in Jilin province, China: occurrence, distribution, and risk assessment. Environ. Earth. Sci., 2016, 75, 1-10.

20. Glanzel, W. and Moed, H. F., Journal impact measures in bibliometric research. Scientometrics, 2002, 53, 171-193.

21. Glanzel, W. and Schoepflin, U., A bibliometric study of reference literature in the sciences and social sciences. Inf. Process Manage., 1999, 35, 31-44.

22. Weingart, P., Impact of bibliometrics upon the science system: inadvertent consequences? Scientometrics, 2005, 62, 117-131.

23. Yin, C. Y., Aris, M. J. and Chen, X., Combination of Eigenfactor ${ }^{\mathrm{TM}}$ and $h$-index to evaluate scientific journals. Scientometrics, 2009, 84, 639-648.

24. Chen, Y. C., Yeh, H.Y., Wu, J. C., Haschler, I., Chen, T. J. and Wetter, T., Taiwan's National Health Insurance Research Database: administrative health care database as study object in bibliometrics. Scientometrics, 2010, 86, 365-380.

25. Tan, J., Fu, H. Z. and Ho, Y. S., A bibliometric analysis of research on proteomics in Science Citation Index Expanded. Scientometrics, 2013, 98, 1473-1490.

26. Xu, Y. Y. and Boeing, W. J., Mapping biofuel field: a bibliometric evaluation of research output. Renew. Sust. Energ. Rev., 2013, 28, 82-91.

27. Mao. N., Wang, M. H. and Ho, Y. S., A bibliometric study of the trend in articles related to risk assessment published in Science Citation Index. Hum. Ecol. Risk Assess., 2010, 16, 801-824.

28. Persson, O. and Dastidar, P. G., Citation analysis to reconstruct the dynamics of Antarctic ozone hole research and formulation of the Montreal Protocol. Curr. Sci., 2013, 104, 835-840.

29. Leydesdorff, L., Bornmann, L. and Wagner, C. S., Generating clustered journal maps: an automated system for hierarchical classification. Scientometrics, 2017, 110, 1601-1614.

30. Zheng, C. L., Cotner, J. B., Sato, C., Li, G. and Xu, Y. Y., Global development of the studies focused on antibiotics in aquatic systems from 1945-2017. Environ. Sci. Pollut. Res., 2018, 25, 22023-22034; doi: 10.1007/s11356-018-2331-5.

31. Björklund, H. V., Råbergh, C. M. I. and Bylund, G., Residues of oxolinic acid and oxytetracycline in fish and sediments from fish farms. Aquaculture, 1991, 97, 85-96.

32. Lalumera, G. M., Calamari, D., Galli, P., Castiglioni, S., Crosa, G. and Fanelli, R., Preliminary investigation on the environmental occurrence and effects of antibiotics used in aquaculture in Italy. Chemosphere, 2004, 54, 661-668.

33. Hektoen, H., Berge, J. A., Hormazabal, V. and Yndestad, M., Persistence of antibacterial agents in marine sediments. Aquaculture, 1995, 133, 175-184.

34. Kemper, N., Veterinary antibiotics in the aquatic and terrestrial environment. Ecol. Indic., 2008, 8, 1-13.

35. Bauer, A. W., Kirby, W. M., Sherris, J. C. and Turck, M., Antibiotic susceptibility testing by a standardized single disk method. Am. J. Clin. Pathol., 1966, 45, 493-496.

36. Mincer, T. J., Jensen, P. R., Kauffman, C. A. and Fenical, W., Widespread and persistent populations of a major new marine actinomycete taxon in ocean sediments. Appl. Environ. Microbiol., 2002, 68, 5005-5011.

37. Bérdy, J., Bioactive microbial metabolites. J. Antibiot., 2005, 58, $1-26$.

38. Lindsey, M. E., Meyer, T. M. and Thurman, E. M., Analysis of trace levels of sulfonamide and tetracycline antimicrobials in groundwater and surface water using solid-phase extraction and liquid chromatography/mass spectrometry. Anal. Chem., 2001, 73, 4640-4646.

39. Chopra, I. and Roberts, M., Tetracycline antibiotics: mode of action, applications, molecular biology, and epidemiology of bacterial resistance. Microbiol. Mol. Biol. Rev., 2001, 65, 232-260.

40. Fenical, W. and Jensen, P. R., Developing a new resource for drug discovery: marine actinomycete bacteria. Nature Chem. Biol., 2006, 2, 666-673.

41. Jacobsen, A. M., Halling-Sørensen, B., Ingerslev, F. and Honoré Hansen, S., Simultaneous extraction of tetracycline, macrolide and sulfonamide antibiotics from agricultural soils using pressurised liquid extraction, followed by solid-phase extraction and liquid chromatography - tandem mass spectrometry. J. Chromatogr. A, 2004, 1038, 157-170.

42. Tolls, J., Sorption of veterinary pharmaceuticals in soils: a review. Environ. Sci. Technol., 2001, 35, 3397-3406.

43. Daughton, C. G. and Ternes, T. A., Pharmaceuticals and personal care products in the environment: agents of subtle change? Environ. Health Perspect., 1999, 107, 907-938.

44. Jiang, Y., Li, M., Guo, C., An, D., Xu, J., Zhang, Y. and Xi, B., Distribution and ecological risk of antibiotics in a typical effluentreceiving river (Wangyang River) in north China. Chemosphere, 2014, 112, 267-274.

45. Liu, H., Zhang, G., Liu, C., Li, L. and Xiang, M., The occurrence of chloramphenicol and tetracyclines in municipal sewage and the Nanming River, Guiyang City, China. J. Environ. Monit., 2009, 11, 1199-1205.

46. Xie, Z., Lu, G., Liu, J., Yan, Z., Ma, B., Zhang, Z. and Chen, W., Occurrence, bioaccumulation, and trophic magnification of pharmaceutically active compounds in Taihu Lake, China. Chemosphere, 2015, 138, 140-147.

47. Zhang, Q. Q., Ying, G. G., Pan, C. G., Liu, Y. S. and Zhao, I. L., Comprehensive evaluation of antibiotics emission and fate in the river basins of China: source analysis, multimedia modeling, and linkage to bacterial resistance. Environ. Sci. Technol., 2015, 49, 6772-6782.

48. Zuccato, E., Calamari, D., Natangelo, M. and Fanelli, R., Presence of therapeutic drugs in the environment. Lancet, 2000, 355, 17891790 .

49. Silva, B. F., Jelic, A., López-Serna, R., Mozeto, A. A., Petrovic, M. and Barceló, D., Occurrence and distribution of pharmaceuticals in surface water, suspended solids and sediments of the Ebro river basin, Spain. Chemosphere, 2011, 85, 1331-1339.

ACKNOWLEDGEMENTS. This work was supported by the Ministry of Science and Technology of China (MSTC) with National Key Research and Development Program (2016YFC0502704), Chinese Academy of Sciences (CAS) with Pioneer Hundred Talents Program (Y7I0021F50), Key Laboratory of Urban Environment and Health Program (KLUEH-C-201701)and National Natural Science Foundation of China (NSFC) with Young Scientists Programs (31200358; 31300397), Ningbo Science and Technology Bureau (NSTB) with Key Scientific and Technological Program (2015C110001). Any opinions, findings, and conclusions or recommendations expressed in this article are those of the author(s), and do not necessarily reflect the views of MSTC, CAS, NSFC and NSTB.

Received 12 July 2018; revised accepted 14 March 2019

doi: $10.18520 / \mathrm{cs} / \mathrm{v} 117 / \mathrm{i} 1 / 37-45$ 